\title{
EVALUACIÓN DE RESULTADOS EN LA ESCUELA NUEVA DE COLOMBIA ¿Es el multigrado la respuesta?
}

\section{George Psacharopoulos \\ Carlos Rojas \\ Eduardo Vélez}

\section{Introducción}

Al igual que muchas otras naciones en desarrollo, Colombia enfrenta el doble reto de mejorar la calidad de la educación primaria en las áreas rurales así como ampliar el cubrimiento y la retención. A pesar del mandato constitucional que establece una educación primaria universal, a mediados del decenio de 1980 el $50 \%$ de las escuelas de las áreas rurales no ofrecían un programa completo de educación primada y $55 \%$ de los niños pertenecientes a esas mismas áreas de edades entre 5 y 7 años $(26 \%$ entre los 10 y los 14 años) jamás habían asistido a la escuela. La eficiencia interna de las escuelas rurales de primaria ${ }^{1}$ fue de menos de $20 \%$, en 1983 mientras que la deserción en el primer año fue de cerca de $35 \%$ en las áreas rurales (véase MOE, 1986).

Originadas en las teorías de la enseñanza multigrado y la promoción automática, las escuelas unitarias, impulsadas por la UNESCO ${ }^{2}$, se establecieron a comienzos del decenio de 1960 en áreas rurales de baja densidad de población. Las características principales de la escuela unitaria son: la presencia de un solo maestro/a (multigrado), la promoción automática, el aprendizaje que permite al niño avanzar a su propio ritmo, los materiales educativos que permiten al maestro/a trabajar con varios grupos a la vez y un/a maestro/a que guía a sus niños/as en lugar de exponer ex cathedra.

Hacia mediados de los 1960, el programa se había ampliado en 150 escuelas piloto de un departamento colombiano. Pero cuando varios años más tarde se hizo un esfuerzo para ampliar su cobertura a todo el país, se presentaron varios problemas (por ejemplo, el entrenamiento de maestros/as, la aplicación del sistema de promoción automática, la relación entre los contenidos de los cursos y el entorno rural en el que vivían los niños/as). En respuesta a estos problemas, se creó la Escuela Nueva a mediados de los 70 como programa oficial del Ministerio de Educación para mejorar aspectos curriculares, de entrenamiento y administrativos en las Escuelas Unitarias.

Desde sus comienzos, la Escuela Nueva se ha expandido rápidamente. Habiendo comenzado en 1976 con unas pocas escuelas, en 1978 ya había más de 500 que participaban en el programa y otras 1500 hasta 1982. Con financiación parcial del Banco Mundial, la Escuela Nueva se extendió una vez más a varios departamentos con un cubrimiento de varios miles de escuelas rurales y para 1989 operaban en el territorio nacional 17.948 que servían a 800.000 estudiantes. Se espera que en los próximos años el programa de la Escuela Nueva cubra todas las áreas rurales (casi 41.000), con el objetivo de incrementar el porcentaje de niños rurales que terminan la primaria de $20 \%$ en 1984 a $60 \%$ aproximadamente.

\footnotetext{
${ }^{1}$ Medido según el porcentaje de estudiantes de primer año que comletaron el quinto año en cinco años.

${ }^{2}$ Recomendación \# 52 de la Conferencia Internacional de Ministros de Educación. Puede leerse una descripción completa de la historia del programa en Colbert de Arboleda (1987).
} 
La Escuela Nueva es una escuela rural en la que uno/a o dos maestros/as enseñan todos los cinco años del ciclo elemental ${ }^{3}$. Difiere de la escuela tradicional en los siguientes aspectos:

- La escuela nueva es multigrado, es decir, uno/a o dos maestros/ as instruyen a los niños/as de diferentes grados, todos en uno o dos salones.

- La Escuela Nueva tiene un sistema de promoción flexible, aunque no automático. El estudiante es promovido al siguiente nivel una vez haya completado un mínimo de objetivos educativos, lo que podría tomar más de una año académico. Este esquema alteró el de promoción automática de la Escuela Unitaria.

- La Escuela Nueva presenta materiales especiales de instrucción, como las guías para el maestro/a y el/la estudiante. El componente curricular consta de guías para los estudiantes y de manuales para los/las maestros/as supervisores. Las guías del estudiante $^{4}$ facilitan el trabajo individual y en grupo. Estas guías complementan los textos y se diseñan de acuerdo el currículo nacional aprobado.

- La Escuela Nueva tiene un currículo orientado al campo. Las guías, desarrolladas por los maestros/as mismos durante sesiones de entrenamiento, ayudan a que el maestro/a adapte el currículo nacional a las necesidades locales, y estimule la aplicación práctica de lo que el estudiante ha aprendido en la escuela a la vida de la comunidad.

- La Escuela Nueva requiere de maestros/as especialmente entrenados en dos áreas 1) contenidos y 2) la aplicación de los diversos materiales educativos del programa. Los componentes de entrenamiento y de seguimiento diseñados para maestros/as y administradores se elaboran en una serie de tres talleres durante los cuales los maestros/ as aprenden a comprometer a la comunidad en el uso de los nuevos materiales educativos, las guías y la biblioteca escolar. El entrenamiento, así como el diseño de los materiales, se lleva a cabo secuencialmente, de modo que los maestros/as puedan aplicar gradualmente lo que aprendan. El entrenamiento se convierte en un proceso activo de aprendizaje sobre cómo poner en práctica el programa.

- La Escuela Nueva apoya el aprender enseñando: los/as estudiantes mayores enseñan a los/as menores (instrucción entre pares).

- La Escuela Nueva tiene rincones de estudio y pequeñas bibliotecas. Los rincones de estudio son áreas establecidas para actividades que se centran en diversos temas de enseñanza, ciencias, matemáticas y sociales. La biblioteca ofrece apoyo no sólo para los estudiantes; es un centro diseñado para la comunidad en general.

- La Escuela Nueva pretende integrar a los estudiantes, las escuelas y la comunidad por medio del estimulo a los maestros/as, a los/as estudiantes y a los padres para que participen en las actividades escolares. Entre los ejemplos de tales actividades se cuentan el mapa del área adyacente a la escuela, los registros familiares, una monografía del municipio y un calendario agrícola. El objetivo consiste en comprometer activamente a los padres para que apoyen el aprendizaje de sus hijos.

\footnotetext{
${ }^{3}$ Se encuentra una descripción completa de la escuela nueva en Schiefelbein (1991).

${ }^{4}$ Las guías describen los objetivos que deben alcanzarse y la información básica y las actividades prácticas para alcanzarlos. Orientan a los estudiantes en las actividades que deben seguir Los estudiantes reciben un paquete de guías que el gobierno les facilita sin costo alguno.

Digitalizado por RED ACADEMICA
} 
- La Escuela Nueva recibe visitas frecuentes de supervisión, que son parte del esfuerzo por dinamizar la administración del programa en el nivel regional, y que incluyen asistencia técnica y orientación para los supervisores.

- El programa incluye además la organización del gobierno estudiantil, uno de los elementos innovadores de la Escuela Nueva. Se estimula a estudiantes, maestros y padres para que participen activamente en las actividades escolares, comprendidas su dirección y su organización.

- Un mecanismo de autocontrol permite que controlen su propia asistencia y comuniquen al maestro/a sus preocupaciones y problemas por medio de la caja de sugerencias. Los maestros/as controlan el progreso de los/las estudiantes utilizando un libro de control de progreso; una vez un/a estudiante termina un ejercicio, debe mostrarlo al maestro/a para su evaluación y para obtener la autorización de iniciar una nueva actividad. Con este libro, los maestros/as controlan el desempeño de los estudiantes en cada módulo, que es parte del concepto de promoción flexible, ya mencionado.

- La Escuela Nueva presenta una escuela de demostración en cada distrito educativo, como ejemplo de cómo debe funcionar una escuela exitosa.

Una escuela tradicional, por supuesto, es aquella que no proporciona atención especial a los estudiantes lentos, y no estimula al estudiante con materiales especiales.

\section{La muestra}

Después de algunos años de puesta en práctica, en 1987 el Ministerio de Educación realizó una evaluación de la Escuela Nueva para determinar el logro cognoscitivo de los/as estudiantes de los grados tercero y quinto en matemáticas, y en español, y además en autoestima, creatividad y conducta cívica. Los datos crudos utilizados en este trabajo son tomados de ese estudio (véase Rojas y Castillo, 1988). Se empleó un diseño cuasi experimental para comparar la Escuela Nueva con las escuelas tradicionales de las áreas rurales de las 12 regiones que la habían experimentado. Estas se escogieron aleatoriamente, tomando en consideración la cantidad de tiempo y la profundidad de la participación en el programa de la Escuela Nueva (por lo menos cinco años y una ejecución completa de los componentes de la Escuela Nueva). Se seleccionaron las escuelas tradicionales de los mismos distritos en los que se habían escogido las de la muestra de la Escuela Nueva. La muestra no está sesgada selectivamente en el sentido de que la Escuela Nueva se ofrece sólo en ciertas áreas rurales del país y los estudiantes que allí viven no tienen realmente otra opción en cuanto a asistir a una u otra escuela.

En conjunto, se hicieron visitas a 168 escuelas de la modalidad nueva y a 60 de la tradicional y se tomó una muestra de 3033 estudiantes. Se dieron pruebas de español y de matemáticas a 1702 estudiantes del grado tercero y a 1331 del quinto (véase Cuadro 1). Estas se complementaron con un conjunto de pruebas de autoestima, creatividad y cívica (conducta democrática), aspectos que se supone son afectados positivamente por la Escuela Nueva. Además, se usaron los cuestionarios para generar información sobre las características de los/as estudiantes, los/as maestros y la escuela. 


\section{Tabla 1}

Distribución por escuela, tipo y grado

$\begin{array}{lccc}\text { Grado } & \text { Tradicional } & \text { Escuela } & \text { Total } \\ \text { Tercero } & 687 & 1.015 & 1.702 \\ \text { Quinto } & 582 & 749 & 1.331 \\ \text { Total } & 1.269 & 1.764 & 3.033\end{array}$

La tabla 2 presenta un resumen de las estadísticas de las variables claves utilizadas en el análisis. Los estudiantes de ambos tipos de escuela eran un poco mayores de lo que podría esperarse en la teoría (10 y 12 años para los grados tercero y quinto, respectivamente), lo que refleja demoras en el ingreso o repetición, fenómenos que se observan particularmente en las áreas rurales del país. De hecho, la mitad de los/ las estudiantes de la muestra habían repetido, en particular los de la escuela tradicional, y casi $40 \%$ había asistido a más de una escuela. Se evidencia la naturaleza rural de la muestra según el tamaño familiar de 7,5 miembros, que se encontró para todos los tipos de estudiantes. (El promedio nacional está cerca de los 5 miembros por familia, según el último censo nacional de 1985.) La muestra se dividió aproximadamente por igual entre muchachos y muchachas en ambos tipos de escuelas y grados. Una de cada tres estudiantes había trabajado para ganar un salario, independientemente del tipo de escuela o de nivel. 
Tabla 2

Media de las variables por escuela, tipo y grado

\begin{tabular}{|c|c|c|c|c|c|}
\hline \multirow[b]{2}{*}{ Variable* } & \multirow{2}{*}{$\begin{array}{c}\text { Grado } \\
\text { Muestra }\end{array}$} & \multicolumn{2}{|c|}{ Tipo Escuela } & \multicolumn{2}{|c|}{ Grado } \\
\hline & & Nueva & Tradicional & Tercero & Quinto \\
\hline \multicolumn{6}{|c|}{$\begin{array}{l}\text { Características } \\
\text { Estudiantiles }\end{array}$} \\
\hline Edad & 11.42 & 11.47 & 11.36 & 10.61 & 12.57 \\
\hline Varón & .52 & .52 & .53 & .52 & .53 \\
\hline TRABAJA & .37 & .36 & .38 & .38 & .36 \\
\hline REPITENTE & .52 & .49 & .55 & .52 & .50 \\
\hline DESERTOR & .11 & .10 & .12 & .06 & .17 \\
\hline OTRAESC & .37 & .38 & .35 & .35 & .39 \\
\hline HORASTV & 1.46 & 1.38 & 1.58 & 1.52 & 1.39 \\
\hline \multicolumn{6}{|c|}{$\begin{array}{l}\text { Características } \\
\text { Familiares }\end{array}$} \\
\hline POBRE & .26 & .22 & .32 & .29 & .21 \\
\hline TAMFAM & 7.45 & 7.48 & 7.41 & 7.48 & 7.42 \\
\hline LIBROS & .78 & .78 & .78 & .80 & .75 \\
\hline TV & .48 & .45 & .51 & .46 & .49 \\
\hline RADIO & .86 & .87 & .84 & .88 & .83 \\
\hline \multicolumn{6}{|c|}{$\begin{array}{l}\text { Características } \\
\text { Escolares }\end{array}$} \\
\hline NUEVA & .58 & 1.00 & .00 & .60 & .56 \\
\hline ESTRATO & 26.00 & 25.81 & 26.28 & 25.80 & 26.30 \\
\hline LUZ & .79 & .77 & .82 & .76 & .83 \\
\hline SUPERV & 3.35 & 3.81 & 2.73 & 3.02 & 3.78 \\
\hline \multicolumn{6}{|c|}{$\begin{array}{l}\text { Características } \\
\text { De los docentes }\end{array}$} \\
\hline FEM & .75 & .74 & .75 & .75 & .74 \\
\hline EDAD & 34.83 & 33.15 & 37.25 & 35.00 & 34.59 \\
\hline EST & 11.81 & 11.84 & 11.78 & 11.75 & 11.90 \\
\hline NORMAL & .74 & .73 & .77 & .79 & .68 \\
\hline UNIV & .12 & .12 & .13 & .15 & .10 \\
\hline EXPT & 13.70 & 12.23 & 15.74 & 13.85 & 13.51 \\
\hline EXPNUEV & 3.58 & 5.47 & .95 & 3.02 & 4.29 \\
\hline EXPEAC & 8.73 & 8.04 & 9.69 & 8.33 & 9.25 \\
\hline MAVIESC & .26 & .33 & .17 & .27 & .25 \\
\hline ESCS & 4.73 & 4.34 & 5.28 & 4.61 & 4.90 \\
\hline
\end{tabular}

* Véanse las definiciones en el apéndice

Más familias de los estudiantes que asistían a la escuela tradicional vivían en áreas rurales deprimidas del país. En relación con otras características familiares, no se observaron diferencias en cuanto a presencia de libros, radio o televisor En relación con las características escolares, la Escuela Nueva tenía mejores indicadores de visitas de supervisión y una razón más alta de estudiante/maestro, aunque tenía menor acceso a la electricidad.

Por lo que hace a las características de los maestros/as, los de las escuelas tradicionales eran mayores en edad y tenían más experiencia, tenían salarios más altos, aunque tenían menos educación formal y menos educación universitaria. En comparación 
con las de Escuela Nueva, las tradicionales probablemente tenían más maestras y más maestros/as que vivían fuera de la escuela. La mayoría de los maestros/as de ambos tipos de escuelas y grados habían estudiado pedagogía durante la secundaria y en la universidad.

\section{Explicación del logro}

Las medias de las puntuaciones por tipo de escuela y grado se presentan en la tabla $3^{5}$. Para facilitar las comparaciones entre los estudiantes de diferentes escuelas, las pruebas se estandarizaron a una media de 50 con una desviación estándar de $10^{6}$. Las medias de las pruebas cognoscitivas revelaron que los/as estudiantes de la Escuela Nueva tuvieron puntuaciones más altas que los/as de la escuela tradicional, excepto en matemáticas en el grado quinto ${ }^{7}$. En cuanto a las pruebas no-congnoscitívas ${ }^{8}$, las puntuaciones promedio fueron otra vez másaltas para los estudiantes de la Escuela Nueva, en especial entre los del grado tercero. En el análisis, también incluimos como una variable de resultado el que un estudiante declarara que él/ella abandonada la escuela al finalizar el año escolar Como era de esperarse, entre los del grado quinto la propensión a abandonar fue mayor Si bien esta propensión era menor entre los estudiantes del grado quinto de la Escuela Nueva, era mayor entre los del tercero.

Tabla 3

\section{Puntuaciones medias por grado y tipo de escuela}

\begin{tabular}{llllll} 
& \multicolumn{3}{c}{ Tercero } & \multicolumn{2}{c}{ Grado } \\
& \multicolumn{2}{c}{ Nueva } & Tradicional & Nueva & Tradicional \\
& 52.3 & 48.6 & n.a. & n.a. \\
\hline ZSCORE(ESPA3) & 52.3 & 48.7 & n.a. & n.a. \\
ZSCORE(MAT3) & 51.1 & n.a. & 50.5 & 49.4 \\
ZSCORE(ESPA5) & n.a. & n.a & 49.9 & 50.1 \\
ZSCORE(MATE5) & n.a. & 46.2 & 54.6 & 54.8 \\
ZSCORE(CRAETIV) & 47.0 & 45.1 & 54.0 & 53.8 \\
ZSCORE(CIVIC) & 48.0 & 48.0 & 51.9 & 51.2 \\
ZSCORE(AUTOEST) & 49.3 & 48.0 & 54.5 \\
REPITENTE (\%) & 50.6 & 55.2 & 47.1 & 20.1 \\
RDRPOUT (\%) & 6.9 & 5.2 & 15.4 &
\end{tabular}

\section{n.a. $=$ no aplicable}

Parece que en las áreas rurales y en las urbanas deprimidas las políticas rígidas de promoción desestimulan a los estudiantes y a los padres. De acuerdo con muchos

\footnotetext{
${ }^{5}$ Se diseñaron varias pruebas para medir el logro, específicas para cada grado. Las pruebas de español comprendieron preguntas abiertas para comprensión de lectura, destreza de escritura y gramática básica de acuerdo con el currículo nacional La confiabilidad de cada prueba fue de 0,82 y 0,86 , repesctivamente. Las pruebas de matemáticas constaban de 20 items cada una. La confiabilidad de la prueba para el grado tercero fue de 0,84 y para la del quinto, de 0,80 .

${ }_{7}^{6}$ La fórmula para la estandarización fue $\mathrm{T}=[10(\mathrm{Xi}-\mathrm{X}) / \mathrm{Sx}]+50$

${ }^{7}$ A pesar de los hallazgos positivos en los estudiantes de la Escuela Nueva, sus puntuaciones indican que sólo alcanzaron un tercio de los objetivos curriculares pretendidos en el caso de las matemáticas para los dos grados y de un poco más de la mitad en los de español en ambos grados.

${ }^{8}$ Se desarrolló un instrumento diferente para cada una de las pruebas no-cognoscitivas. Para la creatividad se diseñó un cuestionario de tres itemes para determinar la flexibilidad, la fluidez y la originalidad. La autoestima se determinó utilizando una escala de Likert, con 26 items y la conducta cívica se determinó con 14 preguntas de selección múltiple. El coeficiente de confiabilidad de estos instrumentos fue de 0,76, 0,84 y 0,71 , respectivamente.
} 
observadores, la combinación de libros de texto y de guías ayuda a los/as estudiantes a orientar su trabajo y deja en mayor libertad al profesor/a para ayudar al/la estudiante desaventajado. Otro resultado benéfico de la Escuela Nueva es que ha incrementado la participación de la comunidad en las actividades educativas y ha estimulado los valores democráticos por medio del mecanismo del gobierno escolar. Resulta estimulante que la Escuela Nueva haga del aprendizaje una empresa no sólo personal sino comunitaria. Por último, de acuerdo con los observadores, la capacitación continuada de los/as maestros/as por medio de este enfoque ha resultado muy benéfico para estimular el aprendizaje.

Las diferencias en las medias crudas, por supuesto, pueden esconder el efecto de los factores individuales sobre el desempeño del estudiante, más allá del hecho de que asista a una Escuela Nueva o a la tradicional. Es necesario disponer de control sobre estos otros factores para poder enunciar conclusiones sobre la efectividad de la Escuela Nueva para mejorar el logro estudiantil. Para este propósito, se ha creado una función de producción educativa estandarizada que explique el logro estudiantil en las escuelas. Las funciones se ajustaron a los estudiantes de los grados tercero y quinto para aquellos que tomaron las pruebas correspondientes de español y de matemáticas y para los que respondieron a los instrumentos de creatividad, autoestima y cívica, con independencia del grado: Logro $=f(t i p o$ de escuela, antecedentes del estudiante, factores escolares, características de los maestros).

\section{Tablas 4 y 5 \\ Puntuaciones promedio por grado en la Escuela Nueva y en la tradicional. Diferencias estadísticamente signflcativa a $P \leq .05$}

Grado 3 Puntuaciones promedio

\begin{tabular}{lccccc} 
& Español & Matem. & Creatividad & Cívica & Autoestima \\
\hline Nueva & 52.3 & 51.1 & 47 & 48 & 49.3 \\
Tradicional & 48.6 & 48.7 & 46.2 & 45.1 & 48
\end{tabular}

Grado 5 Puntuaciones promedio

\begin{tabular}{lccccc} 
& Español & Matem. & Creatividad & Cívica & Autoestima \\
\cline { 1 - 5 } Nueva & 50.5 & 49.9 & 54.6 & 54 & 51.9 \\
Tradicional & 49.4 & 50.1 & 54.8 & 53.8 & 51.2
\end{tabular}

La tabla 5 presenta los resultados de este análisis multivariado para controlar los diversos factores que podrían afectar simultáneamente el logro académico ${ }^{9}$. Los resultados de los estudiantes de tercer grado aparecen en las columnas uno y dos, y para el grado quinto en las columnas 3 y 4 . (La Escuela Nueva es una variable 0-1 nula, en la que cero es la escuela tradicional.) La Escuela Nueva está relacionada positivamente con el logro, salvo en las matemáticas del quinto grado.

Las características de género y de repitencia tuvieron un impacto significativo en los resultados del logro. Los muchachos alcanzaron un mejor logro que las muchachas en matemáticas, pero no en español, en el que ellas se desempeñaron mejor. Otras características asociadas algunas veces con el logro académico, como el trabajo y el ver

\footnotetext{
${ }^{9}$ Puede encontrase un resumen bibliográfico de los factores que afectan el logro en Fuller (1990). 
TV, no tuvieron un efecto significativo (aunque el ver TV afectó negativamente el logro en español en el tercer grado).

Varias características familiares asociadas con el logro académico según la bibliografía demostraron los resultados esperados. Los estudiantes de familias que viven en las regiones más pobres tuvieron un logro significativamente menor; para corroborarlo, los estudiantes de familias que poseían un televisor — señal de riqueza en las áreas ruralestuvieron un logro mejor, particularmente en las pruebas de español. Tener libros en la casa pareció relevante para los estudiantes del tercer grado, pero no para los del quinto. Por último, la ayuda en la casa para hacer las tareas no pareció tener un efecto significativo.

El tercer conjunto de variables se refiere a las características de la escuela, incluyendo el tipo de escuela. Resulta claro que la Escuela Nueva ha tenido un efecto significativo sobre los/as estudiantes de tercer grado en matemáticas y español, pero no sobre los del quinto. La razón estudiantes/maestros tuvo el esperado impacto negativo sobre los del tercer grado, pero no sobre los del quinto. Tener electricidad, el indicador de las facilidades escolares que usamos, tuvo un impacto positivo sobre el logro. Por último, el número de visitas de los/as supervisores tuvo una relación negativa con el logro académico, lo que señala la posibilidad de que los/as supervisores hubieran visitado más escuelas de menor calidad. 
Tabla 5

Explicación del rendimiento educativo en los grados 3 y 5

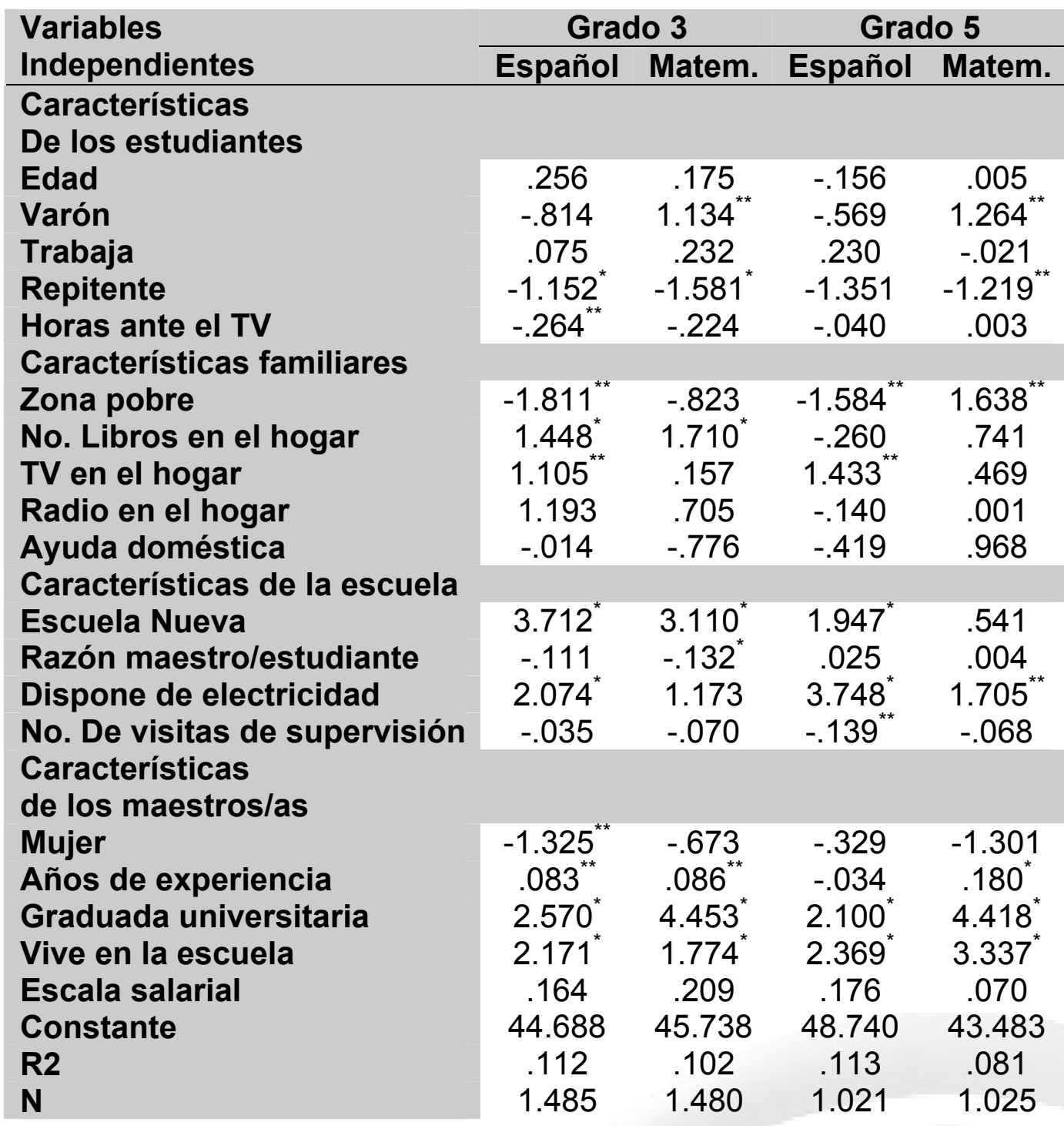

*significancia estadística a $1 \%$ o más

**significancia estadística a $5 \%$

El factor más relevante de los/as maestros/as fue su educación previa al servicio. Un grado universitario tuvo una relación positiva muy alta en todos los puntajes. La experiencia de los maestros/as también se asoció con los resultados más cognoscitivos. Ser maestra no pareció ayudar mucho, en particular en español en el grado tercero. El nivel salarial de los/as maestros no tuvo ningún efecto. Otro factor relevante fue el de residencia del maestro/a; cuando el maestro/a vivía en la escuela, el poder de la interacción maestro/estudiante y el tiempo de la tarea, así como el logro académico de los estudiantes, fue superior al logro cuando el maestro/a vivía en otro lugar.

Los resultados de ambos grados fueron muy semejantes. La única diferencia relevante se encontró en la presencia de libros en el hogar y en la relación estudiante/maestro. El hecho de que los estudiantes del grado quinto tuvieran libros en el hogar no mejoró su logro académico. La otra diferencia muy clara fue que la relación estudiante/maestro afectó negativamente el logro en los estudiantes del grado tercero, pero no en los del quinto. 
La tabla 5 presenta los modelos que se ocupan de la explicación de la creatividad, la conducta cívica y la autoestima. La Escuela Nueva sólo tuvo un efecto independiente sobre la conducta cívica. En la columna 1, los resultados de la creatividad indican que los estudiantes mayores que habían tenido experiencia laboral y no habían repetido ningún año eran más creativos. Entre los factores familiares, el único que arrojó un efecto positivo sobre la creatividad fue tener TV. El impacto negativo que se observó tenían las profesoras sobre el logro académico también se encontró para explicar la creatividad. Los salarios de los/as profesores/as, los años de experiencia y el que el maestro/a viviera en la escuela también se asociaron positivamente con la creatividad.

La conducta cívica, el único elemento no cognoscitivo afectado por la Escuela Nueva, también se explica por las características de los estudiantes, i. e., edad (los mayores tenían más civismo), el género (los muchachos tenían menos), repitencia (los repitentes tenían menos), experiencia laboral (los trabajadores tenían menos), y horas de TV (a más horas de TV, menos civismo).

Tabla 6

Explicación de las puntuaciones no cognoscitivas

\begin{tabular}{|c|c|c|c|}
\hline $\begin{array}{l}\text { Variables } \\
\text { Independientes } \\
\text { Características } \\
\text { De los estudiantes }\end{array}$ & Creatividad & Cívica & Autoestima \\
\hline Edad & $1.307^{*}$ & $1.337^{*}$ & $.889^{*}$ \\
\hline Varón & .140 & $-1.426^{*}$ & $-.919^{* *}$ \\
\hline Trabaja & $.829^{* *}$ & $-.947^{* *}$ & .111 \\
\hline Remitente & $-2.075^{*}$ & $-2.092^{*}$ & $-2.190^{*}$ \\
\hline Horas ante el TV & -.264 & $-.438^{*}$ & -.214 \\
\hline $\begin{array}{l}\text { Características } \\
\text { familiares }\end{array}$ & & & \\
\hline Zona pobre & -.438 & $-1.564^{*}$ & .627 \\
\hline No. Libros en el hogar & .580 & .291 & $2.138^{*}$ \\
\hline TV en el hogar & $2.176^{*}$ & $2.431^{*}$ & .522 \\
\hline Radio en el hogar & .403 & -.055 & -.097 \\
\hline Ayuda doméstica & -.518 & $1.019^{*}$ & .038 \\
\hline $\begin{array}{l}\text { Características } \\
\text { Escolares }\end{array}$ & & & \\
\hline Escuela Nueva & .336 & $1.249^{*}$ & .575 \\
\hline Razón maestro/estudiante & .007 & .014 & -.004 \\
\hline Dispone de electricidad & $2.865^{*}$ & $1.446^{*}$ & $-1.599^{*}$ \\
\hline No. De visitas de supervisión & $.076^{* *}$ & -.006 & $-.086^{\star *}$ \\
\hline $\begin{array}{l}\text { Características } \\
\text { De los/as maestros }\end{array}$ & & & \\
\hline Mujer & $-1.667^{*}$ & .017 & .677 \\
\hline Años de experiencia & $.081^{*}$ & -.015 & -.063 \\
\hline Graduada universitaria & .9821 & $.711^{*}$ & $-3.110^{*}$ \\
\hline Vive en la escuela & $3.144^{*}$ & $1.489^{*}$ & $1.209^{*}$ \\
\hline Escala salarial & $.610^{*}$ & .013 & .099 \\
\hline Constante & 27.633 & 33.019 & 40.709 \\
\hline R2 & .163 & .124 & .067 \\
\hline $\mathbf{N}$ & 2.519 & 2.330 & 2.372 \\
\hline
\end{tabular}

* Estadísticamente significativo en el nivel de 1\% o más.

${ }^{* *}$ Estadísticamente significativo en el nivel de 5\%. 
Entre las características familiares, los estudiantes de las áreas más pobres tenían menos civismo, mientras que los que recibían ayuda para hacer las tareas y veían TV tenían más civismo. Dos hechos relevantes para explicar la conducta cívica tienen que ver con que el maestro/a fuese graduado universitario y tuviera residencia en la escuela.

La autoestima, última columna, se explica débilmente por el modelo. Los varones y los repitentes tenían una autoestima baja en comparación con las mujeres y los no repitentes. Entre las características familiares, la disponibilidad de libros en el hogar fue la única variante que afectó la autoestima, de una manera positiva. En cuanto a las características de la escuela, tener servicio eléctrico y el número de visitas de supervisión parecen haber afectado negativamente la autoestima del estudiante. Los/as maestros que tenían grado universitario tuvieron un impacto negativo sobre la autoestima. Cuando los/as maestros/as vivían en la escuela, la tendencia entre los estudiantes era a un mayor nivel de autoestima.

La tabla 6 presenta otro indicador de la calidad de la escuela: la predicción de la probabilidad de deserción del estudiante. Como ésta es una variable 0-1 limitada, resulta apropiado un modelo logit ${ }^{10}$. La Escuela Nueva redujo significativamente las probabilidades de los estudiantes del grado quinto, pero no las de los de tercero. De acuerdo con los resultados de los estudiantes del grado tercero, la edad y el género eran predictores de la deserción escolar. Los estudiantes mayores varones tuvieron una mayor probabilidad de abandonar el sistema educativo. Para los de grado quinto los resultados fueron un tanto diferentes. Como antes, los muchachos de mayor edad tenían más probabilidad de abandonar el sistema escolar. La edad, empero, presentó una tendencia en la que cada año de edad significaba una probabilidad decreciente de deserción. Para los de grado quinto, la experiencia laboral fue probablemente la característica más significativa de predicción de deserción, algo que debe esperarse en la Colombia rural. Resulta interesante que vivir en un área pobre y la experiencia de repetición no tuvieran un impacto significativo para determinar la probabilidad de deserción autoreportada.

Podría argumentarse que en las áreas de baja población tener o no una escuela multigrado no es una opción. Para este propósito tenemos que repasar las funciones ya enunciadas de las submuestras en el conjunto de la Escuela Nueva para determinar qué factores llevaron a un logro superior en este tipo de escuela. Los resultados (no se presentan en detalle) muestran que una razón maestro/estudiante baja, la disponibilidad de electricidad y un salario más alto, la educación universitaria y vivir en la escuela contribuyeron significativamente a las ganancias en logros.

\footnotetext{
${ }^{10}$ El modelo refleja la probabilidad de que un/a estudiante se retire del sistema escolar el año siguiente, como función de las características seleccionadas.
} 


\section{Tabla 7 \\ Predicción de la probabilidad de deserción por grado}

\begin{tabular}{|c|c|c|}
\hline \multirow{2}{*}{$\begin{array}{l}\text { Variables } \\
\text { Independientes }\end{array}$} & \multicolumn{2}{|c|}{ Coeficientes LOGIT } \\
\hline & $\begin{array}{l}\text { Grado } \\
\text { tercero }\end{array}$ & $\begin{array}{l}\text { Grado } \\
\text { Quinto }\end{array}$ \\
\hline Edad & .139 & -.119 \\
\hline Varón & .546 & .330 \\
\hline Trabaja & .112 & .912 \\
\hline Remitente & -.320 & .036 \\
\hline Área pobre & .061 & -.317 \\
\hline Escuela Nueva & .247 & -.306 \\
\hline Probabilidad log -2 & 776.014 & 991.307 \\
\hline $\mathrm{N}$ & 1,698 & 1,202 \\
\hline Variable dependiente media & 6.2 & 17.4 \\
\hline
\end{tabular}

*Significación estadística en el nivel de $5 \%$ o más.

**Significación estadística en el nivel de $10 \%$.

\section{Conclusiones}

En resumen, nuestros hallazgos indican que la Escuela Nueva ha tenido un efecto independiente significativo sobre los logros de los estudiantes, efectuando control sobre las características del estudiante y sobre las de la familia y los aportes de la escuela.

Otros indicadores que corroboran el resultado positivo de la Escuela Nueva se encuentran en Rojas y Castillo (1988), quienes observaron que a pesar del hecho de que en $42 \%$ de las escuelas de Escuela Nueva no se había organizado el gobierno escolar, estas tenían un grado de participación significativamente más alto medido por actividades como educación de adultos $(35,6 \%$ contra $28,1 \%)$, extensión agrícola (35\% contra $15,8 \%)$, competiciones atléticas $(54,6 \%$ contra $42,5 \%)$, campañas por la salud $(82,9 \%$ contra $56,7 \%)$, y celebraciones de la comunidad (88,5\% contra $83,3 \%)$.

Un punto importante es que los anteriores puntos benéficos se alcanzaron con un costo unitario por estudiante, que no difiere substancialmente de los de la escuela tradicional. Se estima que los costos unitarios de la Escuela Nueva son de $5 \%$ a $10 \%$ más altos que los costos en las escuelas tradicionales (Schiefelbein, 1991). Los costos adicionales de dotar a los estudiantes de guías de estudio, bibliotecas y maestros con un entrenamiento adicional son compensados por el hecho de que la Escuela Nueva sólo tiene uno o dos maestros, en oposición a la situación de un maestro/a por aula de clase de las escuelas tradicionales.

Como precaución, es importante tener presente que hasta tanto no se realice un estudio de costos, los resultados de comparar la Escuela Nueva con la tradicional deben considerarse provisionales. A manera de ejemplo de la implicación de costos, aunque el entrenamiento de un maestro de la Escuela Nueva se enfoca en la enseñanza multigrado (lo que puede tener implicación de ahorro en costos), en la época del entrenamiento se calculó que el costo de la Escuela Nueva era tres veces más alto. De modo que a pesar de los incrementos en el logro y de una mejoría en las tasas de deserción, debe utilizarse la información disponible con cautela cuando se trata de hablar de los beneficios del programa. 
Otro problema que enfrenta la Escuela Nueva es el de la replicabilidad. A pesar de sus logros, se necesitaron quince años para que la Escuela Nueva se convirtiera en un programa formal. Antes de 1982, dependía de dos o tres oficinas del Ministerio de Educación. Hoy, aunque institucionalizada en todo el país, el apoyo que recibe la Escuela Nueva en algunos departamentos depende en gran medida de las preferencias personales de los administradores locales. El carácter conservador del sistema educativo colombiano se ha convertido en un obstáculo para lograr los niveles actuales de ejecución. El apoyo recibido de organizaciones locales privadas (Federación Nacional de Cafeteros y otros) ha resultado ser esencial para el desarrollo y ejecución del programa a escalas piloto y local, pero para una puesta en marcha de un modo masivo en el nivel nacional su papel será menos relevante.

A pesar del apoyo de entidades internacionales (AID, IDB Unicef y el Banco Mundial continúan con su apoyo a la Escuela Nueva), no deja de ser un interrogante qué sucedería durante la expansión de este innovador programa. Una preocupación de importancia es que hasta ahora (durante la fase piloto y el período de operaciones en pequeña escala), funcionarios de la Escuela Nueva altamente motivados han podido controlar la calidad de su ejecución, pero con su expansión masiva actual sería muy difícil conservar la calidad del programa. Es importante seguir la expansión del proceso actual puesto que será la prueba verdadera del proceso relacionado con el entrenamiento de maestros/as, la entrega de materiales y el seguimiento de las escuelas que constituyen el modelo de la Escuela Nueva.

\section{Bibliografía}

Colbert de Arboleda, C. V. "Universalización de la primaria en Colombia: el programa de Escuela Nueva", en La educación rural en Colombia: situación, experiencias y perspectivas, editado por la Fundación para la Educación Superior, FES, Bogotá, 1987.

Fuller, B. "Wbat Investments Raise Achievement in the Third World?" en Improving Educational Quality: A Global Perspective. D. W Chapman y C. A. Carrier (eds). Nueva York: Greenwood Press, 1970, pp. 7-43.

Ministerio de Educación Nacional, Estadísticas de la Educación, 1982-1986. Oficina Sectorial de Planeación Educativa, División de Estadística y Sistemas, Bogotá, 1986.

Rojas, C. y Castillo, Z. "Evaluación del programa Escuela Nueva en Colombia", Instituto SER de Investigación, IFT-133. Procesado, Bogotá, enero de 1988.

Schiefelbein, E. In Search of the School of the XXI Century. Is the Colombian Escuela Nueva the Right Pathfinder? UNESCO/Unicef, Santiago, 1991.

\section{ANEXO}

Variables independientes utilizadas en el análisis

Características estudiantiles

EDAD

VARÓN

TRABAJA

REPITENTE
En años

Nula 1 = varón, $\mathrm{O}=$ mujer

Trabajo remunerado (1, 0 nula)

Experiencia de repetición de al menos un grado (1, 0 nula) 
SDESERTOR

OTRAESC

TVHORAS
Deserción auto-reportada para el año siguiente (1, 0 nula)

Experiencia escolar en otras escuelas

Número de horas de televisión al día

Características familiares

POBRE

TAMFAM

LIBROS

TV

RADIO
Familia vive en área deprimida ( 1,0 nula)

Tamaño de la familia

Número de libros en el hogar

Hay televisor en el hogar ( 1,0 nula)

Hay radio en el hogar ( 1,0 nula)

Características escolares

NUEVA

ESTRATO

LUZ

SUPERV
Estudiante de Escuela Nueva (1, 0 nula)

Razón estudiante-maestro/a

La escuela tiene electricidad ( 1,0 nula)

Número de visitas de supervisión en los dos últimos años

Características del maestro/a

FEM

EDAD

EST

NORMAL

UNIV

EXPT

EXPNUEV

EXPEAC

ESCS
Maestra (1, 0 nula)

Edad del maestro/a

Número de años formales de educación

Estudió pedagogía durante la secundaria ( 1,0 nula)

Se graduó en una universidad (1, O nula)

Número de años de experiencia

Número de años de experiencia en Escuela Nueva

Número de años de enseñanza en esta escuela MAVIESC

Maestro/a vive en la escuela

Escala salarial (escalafón) 